\title{
Fundamental singularities in the theory of water waves with surface tension
}

\author{
P. F. Rhodes-Robinson
}

\begin{abstract}
In this paper the forms are obtained for the harmonic potential functions describing the fundamental wave-source and multipole singularities which pertain to the study of infinitesimal time-harmonic waves on the free surface of water when the effect of surface tension is included. Line and point singularities are considered for both the cases of infinite and finite constant depth of water. The method used is an extension of that which has been used to obtain these potentials in the absence of surface tension.
\end{abstract}

\section{Introduction}

In Thorne [5] a discussion was given of line and point singularities which have since been used extensively in time-harmonic problems in the linearized theory of water waves propagating on the free surface (FS) of water of either infinite or finite constant depth. However, these are suitable only when the assumption is made that, at the FS, the effect of surface tension can be neglected. Recent investigations, in particular Evans [1, 2] and Rhodes-Robinson [4], involve well known problems in surface waves in which the presence of surface tension has now been allowed for. Here we give a discussion of the fundamental line and point singularities, both submerged and in the FS, which have been used in these and which will be required in future investigations of other two-dimensional and axisymmetric time-harmonic problems in the theory of water waves when the effect of surface tension is included. For

Received 18 February 1970. 
two-dimensional motions, the line singularities considered are a wave source and multipole singularities. Restriction is made to symmetric (or 'vertical') multipoles, but the corresponding antisymmetric (or 'horizontal') multipoles can be found similarly. For axisymetric motions, the point singularities considered are multipole singularities. The time-harmonic singularities are described by harmonic potential functions which satisfy a modified FS condition and uniqueness is ensured by requiring that there are only outgoing surface waves in the far field. The method used is basically as in [5] and is valid only for submerged singularities. However, we also give the results for FS singularities to complete the survey.

\section{Statement of the boundary-value problem}

We are concerned with the irrotational motion of water with a FS which is assumed to be an incompressible inviscid fluid, under the action of gravity and surface tension. The motion is time-harmonic with angular frequency $\sigma$ and due to an oscillating singularity in or below the FS which produces outgoing waves at infinity. The nature of the singularity varies: two-dimensional motions will be produced by line singularities, which may be either a wave source or multipoles, and axisymmetric motions by point singularities, which are multipoles. Further, the region of water is of infinite horizontal extent and may be either of infinite depth or else bounded by a bottom at some finite constant depth $h$. In each case, the singularity can be described by a complex-valued harmonic potential function $\phi$ in which a time factor $e^{-i \sigma t}$ is suppressed in the usual way. If we take an origin $O$ in the mean FS and an axis $O y$ pointing down into the fluid, then this potential satisfies a boundary-value problem in which

$$
\nabla^{2} \phi=0
$$

in the fluid region, except at the singularity,

$$
K \phi+\phi_{y}+M \phi_{y y y}=0 \text { on } y=0 \text {, }
$$

where $K=\sigma^{2} / g$ and $M=T / \rho g$ (this linearized FS condition is derived in Evans [2] and $T$ is the surface tension, $\rho$ the density and $g$ the acceleration of gravity; $M=0$ in the absence of surface tension), and 


$$
\phi_{y}=0 \text { on } y=h
$$

when there is a bottom to the fluid region (otherwise $\nabla \phi \rightarrow 0$ as $y \rightarrow \infty$ ). These two boundary conditions to Laplace's equation are specific for each singularity considered. They are supplemented by the two general limiting conditions that $\phi$ behaves like a typical singular harmonic function near the singularity, and represents outgoing waves in the far field. These will be made specific below.

The boundary-value problem is formulated assuming that the singularity is submerged and not in FS, or on the bottom in the case of finite depth. Under these conditions a unique solution will be found for each type of singularity considered, the proofs depending on the use of appropriate integral representations for singular harmonic functions. We first discuss line singularities (Section 3 ) and then point singularities (Section 4). For each type of singularity we first consider infinite depth (where useful alternative forms of solution are available) and then finite depth. The limiting cases as the singularity moves into the FS are also important and will be discussed briefly (Sections 5,6 ).

\section{Submerged line singularities}

Here we define rectangular coordinates $x, y$ by taking a second axis $O x$ in the mean FS. The line singularity is placed at the point $(0, Y)$ without loss of generality, where $Y>0$ for a submerged singularity. We consider only singularities symetric in $x$ - namely, a wave source and vertical multipoles. Also we define cylindrical polar coordinates $\rho, \zeta$ based on the singularity position by the equations $x=p \sin \zeta$, $y-Y=\rho \cos \zeta$ so that the singularities are symmetric in $\zeta$.

Submerged wave source on infinite depth

Here we seek a potential $\phi$ which is harmonic in the half-plane $y>0$, except at $(0, Y)$, satisfies the FS condition with surface tension on $y=0$, and whose gradient tends to zero as $y \rightarrow \infty$; also,

$$
\phi \rightarrow \log \rho \text { as } \rho \equiv\left[x^{2}+(y-Y)^{2}\right]^{\frac{1}{2}} \rightarrow 0,
$$

and 


$$
\phi \rightarrow \text { a multiple of } e^{-k y+i k|x|} \text { as }|\dot{x}| \rightarrow \infty \text {, }
$$

where $k$ is the infinite-depth wave number with surface tension and satisfies the equation

$$
K\left(1+M k^{2}\right)-K=0 .
$$

To ensure that $\phi$ remains bounded at infinity, we also define $\rho^{\prime} \equiv\left[x^{2}+(y+y)^{2}\right]^{\frac{1}{2}}$ and try as the appropriate form of the solution

$$
\phi=\log \frac{p}{p^{\prime}}+\phi^{\prime},
$$

and hence $\phi^{\prime}$ is a regular harmonic function in $y>0$. But $\phi^{\prime}$ is also symmetric in $x$, and $\nabla \phi^{\prime}$ must tend to zero as $y \rightarrow \infty$. Hence we try the integral form

$$
\phi^{\prime}=\int_{0}^{\infty} A(k) e^{-k y} \cos k x d k
$$

where now $A(k)$ must be chosen so that $\phi$ satisfies the FS condition, and represents outgoing waves at infinity.

Now we have the representation, valid for $|x|>0$,

$$
\log \frac{\rho}{\rho^{\prime}}=-2 \int_{0}^{\infty} \frac{e^{-k|x|}}{k} \sin k y \sin k Y d k \text {. }
$$

Hence

$$
\left[\left(K+\frac{\partial}{\partial y}+M \frac{\partial^{3}}{\partial y^{3}}\right) \log \frac{\rho}{\rho^{\prime}}\right]_{y=0}=-2 \int_{0}^{\infty}\left(1-M k^{2}\right) e^{-k|x|} \sin k Y d k \quad(|x|>0) .
$$

But, since $y>0$, we may show that

$$
\int_{0}^{\infty} k^{2 n} e^{-k|x|_{\sin k Y d k}=(-1)^{n}} \int_{0}^{\infty} k^{2 n} e^{-k Y} \cos k x d k \quad(n=0,1, \ldots),
$$

by putting $2 i \sin k Y=e^{i k Y}-e^{-i k Y}$ and rotating the contour in each integral so formed to contours along the positive and negative imaginary axes respectively. Therefore,

$$
\left[\left(K+\frac{\partial}{\partial y}+M \frac{\partial^{3}}{\partial y^{3}}\right) \log \frac{\rho}{\rho^{\top}}\right]_{y=0}=-2 \int_{0}^{\infty}\left(1+M k^{2}\right) e^{-k Y} \cos k x d k .
$$


But

$$
\left[\left(K+\frac{\partial}{\partial y}+M \frac{\partial^{3}}{\partial y^{3}}\right) \phi^{\prime}\right]_{y=0}=-\int_{0}^{\infty}\left[k\left(1+M k^{2}\right)-k\right] A(k) \cos k x d k
$$

and so $\phi$ satisfies the FS condition if

$$
A(k)=\frac{-2\left(1+M k^{2}\right)}{k\left(1+M k^{2}\right)-K} e^{-k Y} \text {. }
$$

This introduces a simple pole at $k=k$ on the real axis in the integral $\phi^{\prime}$ : if we avoid this by making an indentation of contour below this pole, we obtain in the usual notation the potential

$$
\phi=\log \frac{\rho}{\rho^{\prime}}-2 \int_{0}^{\infty} \frac{\left(1+M k^{2}\right) e^{-k(y+Y)}}{k\left(1+M k^{2}\right)-K} \cos k x d k,
$$

which ensures that, lastly, the radiation condition is satisfied. For, by putting $2 \cos k x=e^{i k|x|}+e^{-i k|x|}$ and rotating the contour in each integral into contours in the first and fourth quadrants respectively, so that we must include the residue term at $k=k$ for the first (note that the two remaining complex-conjugate poles are in the second and third quadrants), we obtain the outgoing waves

$$
\phi \rightarrow-2 \pi i \frac{1+M k^{2}}{1+3 M k^{2}} e^{-\kappa(y+Y)+i \kappa|x|} \text { as }|x| \rightarrow \infty .
$$

An alternative form, valid for $|x|>0$, which makes explicit the outgoing waves at infinity is obtained by rotating the contour as above to contours along the positive and negative imaginary axes respectively,

$$
\begin{aligned}
\phi=\log \frac{\rho}{\rho^{\prime}}-2 \int_{0}^{\infty} \frac{\left(1-M k^{2}\right) e^{-k|x|}}{k^{2}\left(1-M k^{2}\right)^{2}+K^{2}}\left[k\left(1-M k^{2}\right) \cos k(y+Y)\right. & -K \sin k(y+Y)] d k \\
& -2 \pi i \frac{1+M k^{2}}{1+3 M k^{2}} e^{-K(y+Y)+i k|x| .}
\end{aligned}
$$

\section{Submerged wave source on finite depth}

Here $\phi$ is harmonic on the strip $0<y<h$, except at $(0, y)$, satisfies the FS condition with surface tension on $y=0$, and the bottom condition on $y=h$; also,

$$
\phi \rightarrow \log \rho \text { as } \rho \rightarrow 0,
$$


and

$$
\phi \rightarrow a \text { multiple of } \cosh _{0}(h-y) e^{i \kappa_{0}|x|} \text { as }|x| \rightarrow \infty \text {, }
$$

where $k_{0}$ is the finite-depth wave number with surface tension and satisfies the equation

$$
\kappa_{0}\left(1+M \kappa_{0}^{2}\right) \tanh \kappa_{0} h-K=0 .
$$

We now try as the appropriate form of the solution

$$
\phi=\log \frac{\rho}{\rho^{\prime}}+\int_{0}^{\infty}[A(k) \cosh k(h-y)+B(k) \sinh k y] \cos k x d k .
$$

Using again the representation, valid for $|x|>0$,

$$
\log \frac{\rho}{\rho^{\prime}}=-2 \int_{0}^{\infty} \frac{e^{-k|x|}}{k} \sin k y \sin k Y d k \text {, }
$$

we have

$$
\begin{aligned}
{\left[\frac{\partial}{\partial y} \log \frac{\rho}{\rho^{\prime}}\right]_{y=h} } & =-2 \int_{0}^{\infty} e^{-k|x|} \cos k h \sin k y d k \quad(|x|>0) \\
& =2 \int_{0}^{\infty} e^{-k h} \sinh k Y \cos k x d k,
\end{aligned}
$$

by putting $2 \cos k h=e^{i k h}+e^{-i k h}$ and rotating the contour in each integral into contours along the positive and negative imaginary axes respectively, and so $\phi$ satisfies the bottom condition if

$$
B(k)=\frac{-2 e^{-k h}}{k \cosh k h} \sinh k Y .
$$

Further, since we found above that

$$
\left[\left(K+\frac{\partial}{\partial y}+M \frac{\partial^{3}}{\partial y^{3}}\right) \log \frac{\rho}{\rho^{\prime}}\right]_{y=0}=-2 \int_{0}^{\infty}\left(1+M k^{2}\right) e^{-k y} \cos k x d k,
$$

it follows that $\phi$ satisfies the FS condition if

$$
A(k)=\frac{-2\left(1+M k^{2}\right)}{k\left(1+M k^{2}\right) \sinh k h-K \cosh k h} \frac{\cosh k(h-Y)}{\cosh k h} \text {. }
$$


This now introduces a simple pole at $k=\kappa_{0}$ on the real axis, below which we make an indentation of contour to obtain the potential

$$
\phi=\log \frac{\rho}{\rho^{\prime}}
$$

$$
-2 \psi_{0}^{\infty}\left(\frac{\left(1+M k^{2}\right) \cosh k(h-y) \cosh k(h-Y)}{k\left(1+M k^{2}\right) \sinh k h-K \cosh k h}+\frac{e^{-k h}}{k} \sinh k y \sinh k Y\right) \frac{\cos k x}{\cosh k h} d k .
$$

This has the outgoing waves

$$
\phi \rightarrow \frac{-4 \pi i\left(1+M \kappa_{0}^{2}\right) \cosh \kappa_{0}(h-y) \cosh \kappa_{0}(h-Y) e^{i \kappa_{0}|x|}}{2 \kappa_{0} h\left(1+M k_{0}^{2}\right)+\left(1+3 M k_{0}^{2}\right) \sinh \left(2 \kappa_{0} h\right)} \text { as }|x| \rightarrow \infty,
$$

obtained similarly as for infinite depth.

Submerged multipoles on infinite depth

Here $\phi$ is harmonic in the half-plane $y>0$, except at $(0, Y)$, satisfies the FS condition on $y=0$, and has zero gradient as $y \rightarrow \infty$; also, since we confine our attention to vertical multipoles,

$$
\phi \rightarrow \frac{\cos (m+1) \zeta}{\rho^{m+1}} \text { as } \rho \rightarrow 0 \quad(m=0,1, \ldots) \text {, }
$$

and

$$
\phi \rightarrow \text { a multiple of } e^{-k y+i k|x|} \text { as }|x|+\infty \text {. }
$$

We try as solution

$$
\phi=\frac{\cos (m+1) \zeta}{\rho^{m+1}}+\int_{0}^{\infty} A(k) e^{-k y} \cos k x d k
$$

and use the representation, valid for $y<y$,

$$
\frac{\cos (m+1) \zeta}{\rho^{m+1}}=\frac{(-1)^{m+1}}{m !} \int_{0}^{\infty} k^{m} e^{-k(Y-y)} \cos k x d k \text {. }
$$

Hence

$$
\left[\left(K+\frac{\partial}{\partial y}+M \frac{\partial^{3}}{\partial y^{3}}\right) \frac{\cos (m+1) \zeta}{\rho^{m+1}}\right]_{y=0}=\frac{(-1)^{m+1}}{m !} \int_{0}^{\infty} k^{m}\left[k\left(1+M k^{2}\right)+K\right] e^{-k y} \cos k x d k
$$

and it follows that $A(k)$ is determined since $\phi$ satisfies the FS 
condition. Hence we obtain the potential

(3.3) $\phi=\frac{\cos (m+1) \zeta}{\rho^{m+1}}+\frac{(-1)^{m+1}}{m !} \psi_{0}^{\infty} \frac{k^{m}\left[k\left(1+M k^{2}\right)+K\right] e^{-k(y+Y)}}{k\left(1+M k^{2}\right)-K} \cos k x d k$.

The alternative form, valid for $|x|>0$, which makes explicit the outgoing waves at infinity is

$$
\begin{array}{r}
\phi=\frac{\cos (m+1) \zeta}{\rho^{m+1}}+r e\left(\frac{i^{m+1}}{m !} \int_{0}^{\infty} \frac{k^{m} e^{-k|x|}}{k^{2}\left(1-M k^{2}\right)^{2}+k^{2}}\left[k\left(1-M k^{2}\right)+i K\right]^{2} e^{i k(y+Y)} d k\right) \\
\quad+\frac{(-1)^{m+1}}{m !} 2 \pi i \kappa^{m+1} \frac{1+M k^{2}}{1+3 M \kappa^{2}} e^{-k(y+Y)+i \kappa|x|} .
\end{array}
$$

Note that we may form a combination of these which is wave-free (i.e. there are no outgoing waves at infinity),

$$
\begin{aligned}
\phi=K \frac{\cos (m+1) \zeta}{\rho^{m+1}}+(m+1) \frac{\cos (m+2) \zeta}{\rho^{m+2}}+M(m+1)(m+2)(m+3) \frac{\cos (m+4) \zeta}{\rho^{m+4}} \\
\quad+\frac{(-1)^{m}}{m !} \int_{0}^{\infty} k^{m}\left[k\left(1+M k^{2}\right)+K\right] e^{-k(y+Y)} \cos k x d k \quad(m=0,1, \ldots) .
\end{aligned}
$$

Submerged multipoles on finite depth

Here $\phi$ is harmonic on the strip $0<y<h$, except at $(0, Y)$, satisfies the FS condition on $y=0$, and the bottom condition on $y=h ;$ also,

$$
\phi \rightarrow \frac{\cos (m+1) \zeta}{\rho^{m+1}} \text { as } \rho \rightarrow 0 \quad(m=0,1, \ldots)
$$

and

$$
\phi \rightarrow \text { a multiple of } \cosh _{0}(h-y) e^{i \kappa_{0}|x|} \text { as }|x|+\infty .
$$

We now try as solution

$$
\phi=\frac{\cos (m+1) \zeta}{\rho^{m+1}}+\int_{0}^{\infty}[A(k) \cosh k(h-y)+B(k) \sinh k y] \cos k x d k \text {. }
$$

Using the representation, valid for $y>Y$, 


$$
\frac{\cos (m+1) \zeta}{\rho^{m+1}}=\frac{1}{m !} \int_{0}^{\infty} k^{m} e^{-k(y-Y)} \cos k x d k
$$

we have

$$
\left[\frac{\partial}{\partial y} \frac{\cos (m+1) \zeta}{\rho^{m+1}}\right]_{y=h}=\frac{-1}{m !} \int_{0}^{\infty} k^{m+1} e^{-k(h-Y)} \cos k x d k
$$

and so $B(k)$ is determined since $\phi$ satisfies the bottom condition. Further, since we found above that

$$
\left[\left(K+\frac{\partial}{\partial y}+M \frac{\partial^{3}}{\partial y^{3}}\right) \frac{\cos (m+1) \zeta}{\rho^{m+1}}\right]_{y=0}=\frac{(-1)^{m+1}}{m !} \int_{0}^{\infty} k^{m}\left[k\left(1+M k^{2}\right)+K\right] e^{-k Y} \cos k x d k,
$$

it follows that $A(k)$ is determined also since $\phi$ satisfies the FS condition. Hence we obtain the potential

$$
\phi=\frac{\cos (m+1) \zeta}{\rho^{m+1}}+\frac{1}{m !} \psi_{0}^{\infty} \frac{(-1)^{m+1} k^{m} P}{k\left(1+M k^{2}\right) \sinh k h-K \cosh k h} \cos k x d k
$$

where

$$
P=\left[k\left(1+M k^{2}\right)+K\right] e^{-k Y} \cosh k(h-y)+e^{-k(h-Y)}\left[k\left(1+M k^{2}\right) \cosh k y-K \sinh k y\right],
$$

and the contour is indented below the simple pole $k=\kappa_{0}$ to give the outgoing waves

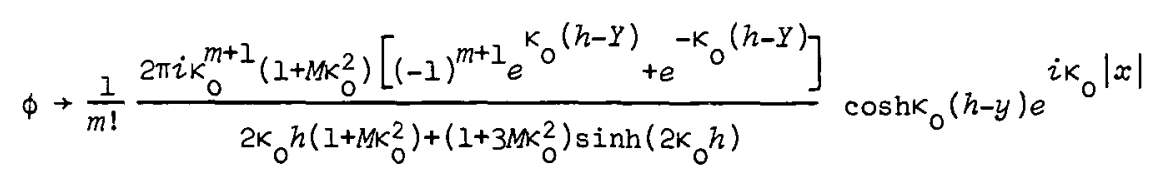

as $|x| \rightarrow \infty$.

\section{Submerged point singularities}

We now define cylindrical polar coordinates $R, y$, where $R$ is the distance from $O y$. The point singularity is placed at the point $(0, Y)$, where $Y>0$. We consider only singularities for which $O y$ is an axis of symetry. Also we define spherical polar coordinates $\rho, \zeta$ based on the singularity position by the equations $R=\rho \sin \zeta$, $y-Y=\rho \cos \zeta$. 
Submerged multipoles on infinite depth

Here $\phi$ is harmonic in the half-plane $y>0$, except at $(0, Y)$, satisfies the FS condition on $y=0$, and has zero gradient as $y \rightarrow \infty$; also,

$$
\phi \rightarrow \frac{P_{m}(\cos \zeta)}{\rho^{m+1}} \text { as } \rho \equiv\left[R^{2}+(y-Y)^{2}\right]^{\frac{1}{2}} \rightarrow 0 \quad(m=0,1, \ldots),
$$

and

$$
\phi \rightarrow \text { a multiple of } e^{-\kappa y_{0}(1)}(K R) \text { as } R \rightarrow \infty \text {. }
$$

We try as solution

$$
\phi=\frac{P_{m}(\cos \zeta)}{\rho^{m+1}}+\int_{0}^{\infty} A(k) e^{-k y_{J}}(k R) d k,
$$

and use the representation, valid for $y<Y$,

$$
\frac{P_{m}(\cos \zeta)}{\rho^{m+1}}=\frac{(-1)^{m}}{m !} \int_{0}^{\infty} k^{m} e^{-k(Y-y)} J_{0}(k R) d k \text {. }
$$

Hence

$$
\left[\left(K+\frac{\partial}{\partial y}+M \frac{\partial^{3}}{\partial y^{3}}\right) \frac{P_{m}(\cos \zeta)}{\rho^{m+1}}\right]_{y=0}=\frac{(-1)^{m}}{m !} \int_{0}^{\infty} k^{m}\left[k\left(1+M k^{2}\right)+K\right] e^{-k Y_{0}} J_{0}(k R) d k
$$

and it follows that $A(k)$ is determined since $\phi$ satisfies the FS condition. Hence we obtain the potential

$$
\text { (4.1) } \phi=\frac{P_{m}(\cos \zeta)}{\rho^{m+1}}+\frac{(-1)^{m}}{m !} \psi_{0}^{\infty} \frac{k^{m}\left[k\left(1+M k^{2}\right)+K\right] e^{-k(y+Y)}}{k\left(1+M k^{2}\right)-K} J_{0}(k R) d k \text {, }
$$

where the contour is indented below the simple pole $k=k$, which ensures that the radiation condition is satisfied. For, by putting $2 J_{0}(k R)=H_{0}^{(1)}(k R)+H_{0}^{(2)}(k R)$ and rotating the contour in each integral into contours in the first and fourth quadrants respectively (where $H_{0}^{(1)}, H_{0}^{(2)}(k R)$ are respectively small), and including the residue term at $k=k$ for the first, we obtain the outgoing cylindrical waves 


$$
\phi+\frac{(-1)^{m}}{m !} 2 \pi i \kappa^{m+1} \frac{1+M k^{2}}{1+3 M k^{2}} e^{-\kappa(y+Y)} H_{0}^{(1)}(K R) \text { as } R \rightarrow \infty .
$$

An alternative form, valid for $R>0$, which makes explicit the outgoing waves at infinity is obtained by rotating the contour to contours along the positive and negative imaginary axes respectively,

$$
\begin{array}{r}
\phi=\frac{P_{m}(\cos \zeta)}{\rho^{m+1}}+r e\left(\frac{i^{m}}{m !} \frac{2}{\pi} \int_{0}^{\infty} \frac{k^{m} K_{0}(k R)}{k^{2}\left(1-M k^{2}\right)^{2}+K^{2}}\left[k\left(1-M k^{2}\right)+i k\right]^{2} e^{i k(y+Y)} d k\right) \\
+\frac{(-1)^{m}}{m !} 2 \pi i K^{m+1} \frac{1+M k^{2}}{1+3 M k^{2}} e^{-k(y+Y)} H_{0}^{(1)}(k R) .
\end{array}
$$

Note that we may form a combination of these which is wave-free,

$$
\begin{aligned}
\phi & =K \frac{P_{m}(\cos \zeta)}{\rho^{m+1}}+(m+1) \frac{P_{m+1}(\cos \zeta)}{\rho^{m+2}}+M(m+1)(m+2)(m+3) \frac{P_{m+3}(\cos \zeta)}{\rho^{m+4}} \\
& +\frac{(-1)^{m+1}}{m !} \int_{0}^{\infty} k^{m}\left[k\left(1+M k^{2}\right)+K\right] e^{-k(y+Y)} J_{0}(k R) d k \quad(m=0,1, \ldots) .
\end{aligned}
$$

Submerged multipoles on finite depth

Here $\phi$ is harmonic on the strip $0<y<h$, except at $(0, Y)$, satisfies the FS condition on $y=0$, and the bottom condition on $y=h ;$ also,

$$
\phi \rightarrow \frac{P_{m}(\cos \zeta)}{\rho^{m+1}} \text { as } \rho \rightarrow 0 \quad(m=0,1, \ldots),
$$

and

$$
\phi \rightarrow \text { a multiple of } \cosh _{0}(h-y) H_{0}^{(l)}\left(\kappa_{0} R\right) \text { as } R \rightarrow \infty \text {. }
$$

We now try as solution

$$
\phi=\frac{P_{m}(\cos \zeta)}{\rho^{m+1}}+\int_{0}^{\infty}[A(k) \cosh k(h-y)+B(k) \sinh k y] J_{0}(k R) d k .
$$

Using the representation, valid for $y>Y$,

$$
\frac{P_{m}(\cos \zeta)}{\rho^{m+1}}=\frac{1}{m !} \int_{0}^{\infty} k^{m} e^{-k(y-Y)} J_{0}(k R) d k \text {, }
$$


we have

$$
\left[\frac{\partial}{\partial y} \frac{P_{m}(\cos \zeta)}{\rho^{m+1}}\right]_{y=h}=\frac{-1}{m !} \int_{0}^{\infty} k^{m+1} e^{-k(h-Y)} J_{0}(k R) d k
$$

and so $B(k)$ is determined since $\phi$ satisfies the bottom condition. Further, since we found above that

$$
\left[\left(K+\frac{\partial}{\partial y}+M \frac{\partial^{3}}{\partial y^{3}}\right) \frac{P_{m}(\cos \zeta)}{\rho^{m+1}}\right]_{y=0}=\frac{(-1)^{m}}{m !} \int_{0}^{\infty} k^{m}\left[k\left(1+M k^{2}\right)+k\right] e^{-k Y_{J}}(k R) d k,
$$

it follows that $A(k)$ is determined also since $\phi$ satisfies the FS condition. Hence we obtain the potential

(4.3) $\phi=\frac{P_{m}(\cos \zeta)}{\rho^{m+I}}+\frac{1}{m !} \int_{0}^{\infty} k^{m} \frac{(-1)^{m} P}{k\left(1+M k^{2}\right) \sinh k h-K \cosh k h} J_{0}(k R) d k$, where again

$$
\left.\left.P=\left[k\left(1+M k^{2}\right)+K\right] e^{-k Y} \cosh k(h-y)+e^{-k(h-Y)}\right] k\left(1+M k^{2}\right) \cosh k y-K \sinh k y\right],
$$

and the contour is indented below the simple pole $k=\kappa_{0}$ to give the outgoing waves

$$
\phi \rightarrow \frac{1}{m !} \frac{2 \pi i \kappa_{0}^{m+1}\left(1+M \kappa_{0}^{2}\right)\left[(-1)^{m_{0} k_{0}(h-Y)}+e^{-\kappa_{0}(h-Y)}\right]}{2 \kappa_{0} h\left(1+M \kappa_{0}^{2}\right)+\left(1+3 M \kappa_{0}^{2}\right) \sinh \left(2 \kappa_{0} h\right)} \cosh \kappa_{0}(h-y) H_{0}^{(I)}\left(\kappa_{0} R\right)
$$

as $R \rightarrow \infty$, obtained similarly as for infinite depth.

\section{FS line singularities}

Clearly the results of Section 3 are not valid for $y=0$. We now define cylindrical polar coordinates $r, \theta$ based on the singularity position by the equations $x=r \sin \theta, y=r \cos \theta$ so that the potentials are symmetric in $\theta$. Then it may be shown that the potentials, where they exist, are as follows.

FS wave source on infinite depth

$$
\phi=-\psi_{0}^{\infty} \frac{\left(1+M k^{2}\right) e^{-k y}}{k\left(1+M k^{2}\right)-K} \cos k x d k \quad(y>0) \text {. }
$$


FS wave source on finite depth

$$
\phi=-\psi_{0}^{\infty} \frac{\left(1+M k^{2}\right) \cosh k(h-y)}{k\left(1+M k^{2}\right) \sinh k h-K \cosh k h} \cos k x d k \quad(y>0) .
$$

FS multipoles on infinite depth

The only genuine multipoles correspond to $m=1,3, \ldots$ ('even' multipoles)

(5.3) $\phi=\frac{1}{(2 n+1) !} \psi_{0}^{\infty} \frac{k^{2 n+2}\left(1+M k^{2}\right) e^{-k y}}{k\left(1+M k^{2}\right)-K} \cos k x d k \quad(n=0,1, \ldots ; y>0)$. Multipoles corresponding to $m=0,2, \ldots$ ('odd' multipoles) do not exist, but in their place there are the singular potentials

$$
\phi=\frac{-K}{(2 n) !} \psi_{0}^{\infty} \frac{k^{2 n} e^{-k y}}{k\left(1+M k^{2}\right)-K} \cos k x d k
$$

$$
\text { ( } n=0,1, \ldots ; y>0 \text { unless } n=0 \text { ) }
$$

which compose the wave source and even multipoles.

The wave-free potentials are

$$
\phi=K \frac{\cos (2 n+1) \theta}{r^{2 n+1}}+(2 n+1) \frac{\cos (2 n+2) \theta}{r^{2 n+2}}
$$

$$
+M(2 n+1)(2 n+2)(2 n+3) \frac{\cos (2 n+4) \theta}{r^{2 n+4}}(n=0,1, \ldots) \text {. }
$$

FS multipoles on finite depth

Similarly we have the even multipoles (for $n=0,1, \ldots$ ),

$$
\phi=\frac{1}{(2 n+1) !} \int_{0}^{\infty} \frac{k^{2 n+2}\left(1+M k^{2}\right) \cosh k(h-y)}{k\left(1+M k^{2}\right) \sinh k h-k \cosh k h} \cos k x d k \quad(y>0)
$$

and the singular potentials

(5.7) $\phi=\frac{-K}{(2 n) !} \psi_{0}^{\infty} \frac{k^{2 n} \cosh k(h-y)}{k\left(1+M k^{2}\right) \sinh k h-K \cosh k h} \cos k x d k \quad(y>0$ unless $n=0)$

which compose the wave source and even multipoles.

For singularities on infinite depth above, alternative forms may be obtained using 


$$
\begin{gathered}
\psi_{0}^{\infty} \frac{k^{2 n} e^{-k y}}{k\left(1+M k^{2}\right)-K} \cos k x d k=(-1)^{n} \int_{0}^{\infty} \frac{k^{2 n} e^{-k|x|}}{k^{2}\left(1-M k^{2}\right)^{2}+K^{2}}\left[k\left(1-M k^{2}\right) \cos k y-K \sin k y\right] d k \\
\quad+\pi i \frac{k^{2 n}}{1+3 M k^{2}} e^{-k y+i k|x|} \quad(n=0,1, \ldots ; \quad|x|>0 \text { unless } n=0) .
\end{gathered}
$$

\section{FS point singularities}

Clearly also the results of Section 4 are not valid for $y=0$. We now define spherical polar coordinates $r, \theta$ based on the singularity position by the equations $R=r \sin \theta, y=r \cos \theta$. Then it may be shown that the potentials are as follows.

FS multipoles on infinite depth

The only genuine (even) multipoles correspond to $m=0,2, \ldots$ (for $n=0,1, \ldots)$

$$
\phi=\frac{1}{(2 n) !} \int_{0}^{\infty} \frac{k^{2 n+1}\left(1+M k^{2}\right) e^{-k y}}{k\left(1+M k^{2}\right)-K} J_{0}(k R) d k \quad(y>0)
$$

since (odd) multipoles corresponding to $m=1,3, \ldots$ do not exist, being replaced by the singular potentials

$$
\phi=\frac{-K}{(2 n+1) !} \int_{0}^{\infty} \frac{k^{2 n+1} e^{-k y}}{k\left(1+M k^{2}\right)-K} J_{0}(k R) d k \quad(y>0 \text { unless } n=0)
$$

which compose the even multipoles.

Alternative forms may be obtained using

$$
\begin{aligned}
& \psi_{0}^{\infty} \frac{k^{2 n+1} e^{-k y}}{k\left(1+M k^{2}\right)-K} J_{0}(k R) d k=(-1)^{n} \frac{2}{\pi} \int_{0}^{\infty} \frac{k^{2 n+1} K_{0}(k R)}{k^{2}\left(1-M k^{2}\right)^{2}+K^{2}}\left[k\left(1-M k^{2}\right) \cos k y-K \sin k y\right] d k \\
& \quad+\pi i \frac{k^{2 n+1}}{1+3 M k^{2}} e^{-K y_{H}(1)}(k R) \quad(n=0,1, \ldots ; R>0 \text { unless } n=0) .
\end{aligned}
$$

The wave-free potentials are

$$
\phi=K \frac{P_{2 n+1}(\cos \theta)}{r^{2 n+2}}+(2 n+2) \frac{P_{2 n+2}(\cos \theta)}{r^{2 n+3}}
$$

$$
+M(2 n+2)(2 n+3)(2 n+4) \frac{P_{2 n+4}(\cos \theta)}{r^{2 n+5}}(n=0,1, \ldots) .
$$


FS multipoles on finite depth

Similarly we have the even multipoles (for $n=0,1, \ldots$ )

$$
\phi=\frac{1}{(2 n) !} \int_{0}^{\infty} \frac{k^{2 n+1}\left(1+M k^{2}\right) \cosh k(h-y)}{k\left(1+M k^{2}\right) \sinh k h-K \cosh k h} J_{0}(k R) d k \quad(y>0)
$$

and the singular potentials

$$
\phi=\frac{-K}{(2 n+1) !} \oint_{0}^{\infty} \frac{k^{2 n+1} \cosh k(h-y)}{k\left(1+M k^{2}\right) \sinh k h-K \cosh k h} J_{0}(k R) d k
$$

$$
(y>0 \text { unless } n=0)
$$

which compose the even multipoles.

\section{Conclusion}

The required wave-source and multipole potentials obtained herein are uniform in the parameter $M$ and known results in the absence of surface tension can be made evident by putting $M=0$. The line singularities considered are symmetric, but those which are antisymmetric can be obtained similarly. Also, the point singularities considered are axisymmetric, but those which are more general can be obtained following Thorne [5].

It should be noted that the FS wave-source and multipole singularities (where they exist) are not unique, since weaker singularities can be added. This cannot occur for the submerged singularities, since the integral terms are regular at the singularity.

Lastly, the wave-free potentials may also be obtained by appropriate use of the following theorem.

THEOREM. If $\Phi$ is a harmonic potential function which is zero on $y=0$, then $\phi=K \Phi-\Phi_{y}-M \Phi_{y y y}$ is a harmonic potential function which satisfies the FS condition with surface tension on $y=0$.

The proof is straightforward.

In the few surface tension problems considered to date, only symmetric line singularities have been used. FS singularities on infinite depth have been employed in Evans [2], an investigation into the motion due to a half-immersed circular cylinder heaving on water of 
infinite depth, where the velocity potential can be expressed in terms (for $n=0,1,2$ ) of the singular potentials (5.4) which compose the wave source and even multipoles, and the wave-free potentials (5.5); and also in Packham [3], the problem of incoming waves on water of infinite depth in the presence of a vertical cliff, where the solution obtained for the velocity potential (in the alternative form which makes explicit the incoming waves) is simply a multiple of the weakest (for $n=0$ ) of the singular potentials (5.4) which compose the wave source and even multipoles (note that this remains bounded at the singularity) but with the indentation of contour now above the simple pole. Submerged singularities on both infinite and finite depth have been employed in Rhodes-Robinson [4], a discussion of the vertical wave-maker problem first investigated in the absence of surface tension by Sir Thomas Havelock, where the solution for the velocity potential is obtained by means of the wave sources $(3.1,3.2)$ respectively.

No use has yet been made of point singularities. However, in their survey article Wehausen and Laitone [6] do give the potential of the first (for $m=0$ ) of the submerged multipoles $(4.1,4.3$ ) on both infinite and finite depth (but without proof).

\section{References}

[1] D.V. Evans, "The influence of surface tension on the reflection of water waves by a plane vertical barrier", Proc. Combridge Phizos. Soc. 64 (1968), 795-810.

[2] D.V. Evans, "The effect of surface tension on the waves produced by a heaving circular cylinder", Proc. Cambridge Philos. Soc. 64 $(1968), 833-847$.

[3] B.A. Packham, "Capillary-gravity waves against a vertical cliff", Proc. Cambridge Phizos. Soc. 64 (1968), 827-832.

[4] P.F. Rhodes-Robinson, "On the forced surface waves due to a vertical wave-maker in the presence of surface tension", (to appear).

[5] R.C. Thorne, "Multipole expansions in the theory of surface waves", Proc. Combridge Phizos. Soc. 49 (1953), 707-716. 
[6] John V. Wehausen and Edmund V. Laitone, "Surface waves", Handbuch der Physik 9, Part 3, 446-778. (Springer-Verlag, Berlin, Göttingen, Heidelberg, 1960).

Victoria University of Wellington, Wellington, New Zealand. 